УДК 616.665-002.28

DOI: $10.26435 /$ UC.V0I3(36).232

\author{
Ю.Ю. Малинин, С.Г. Ермилов, Г.И. Ермилов
}

ГОО ВПО «Донецкий национальный медицинский университет имени М. Горького», Донецк

\title{
ХИРУРГИЧЕСКИЕ МЕТОДЫ ЛЕЧЕНИЯ БОЛЕЗНИ ПЕЙРОНИ
}

Болезнь Пейрони (БП), или фибропластическая индурация полового члена - это заболевание неясной этиологии, при котором на белочной оболочке полового члена возникают фибропластические бляшки, нарушающие ее эластичность и приводящие к эректильной деформации полового члена [1].

Во многих ранних публикациях указывалось, что встречаемость симптоматической БП колеблется от 0,3 до $1 \%$, но согласно современным данным, она составляет от 3 до 10\% [2, 3]. Возможно, это связано с появлением современных методов консервативной терапии эректильной дисфункции (ЭД) и андрогенного дефицита: частота БП растет по мере увеличения возраста, а лечение ЭД у пациентов старшего возраста позволило многим из них начать вести активную половую жизнь, вне которой симптомы БП практически не проявляются [4].

Типичная клиническая картина заболевания представлена триадой симптомов - боль, деформация полового члена при эрекции и зона уплотнения белочной оболочки и межкавернозной перегородки, которая обычно отчетливо определяется при пальпации. Несмотря на то, что это заболевание вызывает озабоченность и настороженность пациентов, оно никогда не угрожает жизни, а нарушение копулятивной функции всегда является основной, снижающей качество жизни жалобой, определяющей потребность в получении медицинской помощи [5].

Существенная деформация полового члена значительно снижает качество жизни пациента и требует хирургического лечения [6,7].

Хирургическое лечение БП на сегодняшний день остается наиболее надежным и быстрым способом устранения девиации полового члена [8]. Несмотря на значительные успехи реконструктивной хирургии полового члена при БП, анализ отечественных и зарубежных источников литературы свидетельствует о неудовлетворительных результатах хирургического лечения, поскольку осложнения и рецидивы болезни, как и раньше, остаются на достаточно высо- ком уровне - от $10 \%$ до $70 \%$ наблюдений $[8,9]$. На сегодняшний день не существует «золотого стандарта» в хирургическом подходе. Однако, главной целью хирургического лечения является выпрямление полового члена и восстановление проникающей способности для адекватного проведения полового акта [10].

Хирургические методы лечения выполняются только в стабильной фазе БП при длительности заболевания не менее 12 месяцев и длительности периода стабилизации деформации пениса и отсутствия боли не менее 6 месяцев [11]. Предложена следующая классификация видов оперативного лечения:

1) укорачивающие методики: со вскрытием белочной оболочки (операция Nesbit, Nesbit-Saalfeld, Nesbit-Lemberger, Nesbit-Hellstrom, Nesbit-Yachia) и без вскрытия белочной оболочки (пликирующие методики - операция NesbitЩеплева, Essed-Schroder, Nesbit-Lue;

2) иссечение (рассечение) бляшек и графтинг с использованием: аутотрансплантатов (венозная стенка, фрагмент фасции, слизистая оболочка щеки), аллотрансплантатов (трупный перикард), ксенотрансплантатов (тонкокишечная субмукоза животных), синтетических гетеротрансплантатов (гортекс, дексон, силастик);

3) имплантация протезов полового члена с графтингом или без [11-15].

Принятие решения о выборе метода оперативного лечения, должно быть адаптировано к ожиданиям конкретного пациента. Важную роль в выборе варианта хирургического лечения играет личный опыт хирурга. Показаниями к хирургическому лечению болезни Пейрони являются: стабильная стадия заболевания как минимум 1 год, стабильная деформация полового члена, отсутствие возможности проведения полового акта, отсутствие эффекта от консервативной терапии, наличие кальцинированной бляшки, желание пациента в стабильной ста-

(c) Ю.Ю. Малинин, С.Г. Ермилов, Г.И. Ермилов, 2020 (c) Университетская Клиника, 2020 
дии, достичь скорейшей коррекции деформации. Во многом, тактика лечения в стабильной стадии зависит от наличия или отсутствия эректильной дисфункции, эффективности ее лечения при медикаментозной терапии, эректильного статуса пациента, угла искривления, наличия сложных форм, укорочения полового члена. $[11,13,14]$. Решающими факторами при выборе того или иного вмешательства являются угол искривления, тип деформации, длина пениса, состояние эректильной функции, а также ожидания пациента и полового партнера. Общепринято, что укорочение белочной оболочки подходит мужчинам с углом искривления до $60^{\circ}$, адекватной длиной и относительно простой конфигурацией полового члена. Операции с замещением участка белочной оболочки должны выполняться, когда угол искривления более $60^{\circ}$, малом размере полового члена, сложной деформации и наличии эффекта «песочных часов». При ЭД целесообразно рассматривать в первую очередь операции с одновременной установкой пенильного имплантата $[12,16]$.

Первая хирургическая операция по удалению бляшки при болезни Пейрони была выполнена George McClellan в 1827 году. В 1928 году было опубликовано о 32 успешных случаях оперативного лечения [17]. В 1965 году Reed Nesbit описал коррекцию врожденной деформации полового члена путем выполнения эллипсовидных разрезов и наложения пликационных швов на противоположной стороне [18]. Впервые данную методику для лечения пациентов с БП в 1979 г. использовали Pryor и Fitzpatrick [19]. Операция Nesbit обычно используется в различных модификациях (эллипсовидное иссечение белочной оболочки, комбинация эллипсовидного иссечения с пликацией, либо пликация белочной оболочки), но все с тем же одним принципом - укорочение противоположной искривлению стороны полового члена.

Данная методика имеет ряд серьезных рисков, связанных с угрозой кровотечения после нарушения целостности белочной оболочки, повреждением уретры и сосудисто-нервного пучка. В послеоперационном периоде довольно высок риск развития кавернита с последующим образованием рубцов в кавернозных телах, что, в свою очередь, приводит к ЭД. Также, возможно снижение чувствительности головки полового члена, гематомы, задержка мочи. Основное осложнение, точнее, негативное последствие операции Nesbit - укорочение полового члена. Тем не менее, оригинальная методика Nesbit до сих пор имеет большую популярность [20]. Авторы публикаций, посвященных оценке результатов применения операции Nesbit в долгосроч- ной перспективе (7-16 лет), отмечают устранение деформации в 80-100\% случаев, достаточное восстановление эрекции в 90,5\%, а порядка 76,2\% пациентов полностью удовлетворены результатами лечения [20].

Укорачивающие операции (операция Nesbit и другие пликационные методы пластики белочной оболочки) являются наиболее простыми, с хорошими результатами, но приводящими к укорочению полового члена. Степень укорочения прямо пропорциональна тяжести исходной деформации, но без ухудшения эректильной функции. Показаниями к пликационной корпоропластике являются: достаточная ригидность полового члена для проведения полового акта (с или без фармакотерапии), сохранная эректильная функция, простая кривизна менее $60^{\circ}$, адекватная длина полового члена, отсутствие деформации по типу «песочных часов» или «шарнирного» искривления, возможная послеоперационная потеря длины менее 20\% от общей длины эрегированного [21].

В начале 1980-х годов были разработаны прогрессивные модификации операции Nesbit без нарушения целостности белочной оболочки - так называемые пликирующие методики. Это было определенным прорывом, так как значительно снижались риски, связанные с кровотечением, а также сокращалась длительность хирургического вмешательства. Основные пликирующие методики было последовательно предложены Щеплевым П.А. в 1983 г., Essed Е. и Schroder F.H. в 1985 г. Общая суть методов состоит в инвагинации белочной оболочки без вскрытия кавернозных тел с использованием не рассасывающегося шовного материала (вворачивающие швы по Essed и Schroder). При этом собственно бляшку не иссекают, с целью профилактики рецидива девиации и дополнительного рубцевания [6].

Результаты модифицированных методов на основе операции Nesbit, с периодом наблюдения 24-72 месяцев, показали успешное выпрямление полового члена у 85-99\% пациентов, с развитием эректильной дисфункции (ЭД) в 3-12\%, и снижением чувствительности у 2-36\% [22].

Основным осложнением, связанным с пликацией при использовании нерассасывающихся нитей, являются гранулемы, формирующиеся на концах нитей, оставленных после их обрезания; в результате чего гранулемы в виде плотных образований пальпируются под кожей [23]. Это побудило Gholami и Lue в 2002 г. применить новую технику 16-dot (16-точечный метод пликации) - накладывали параллельные парные швы, тем самым равномерно распределяя суммарную силу натяжения во время эрекции, а 
также предотвращая разрыв швов. При этой методике была допущена возможность использования рассасывающегося шовного материала [24, 25].

Негативным моментом является низкая повторяемость результатов. Большинство модификаций применяются только самими авторами, независимая оценка, обязательная для медицины, основанной на доказательствах, отсутствует. То есть, обоснованных данных, свидетельствующих о явном преимуществе какойлибо методики, в настоящее время нет. При выборе конкретного вида вмешательства хирург, фактически, руководствуется собственным клиническим опытом [26].

Устранить искривление полового члена без его укорочения позволяют пластические операции: заместительная корпоропластика или графтинг, а дефект белочной оболочки после иссечения бляшки закрывают трансплантатом [5]. Методом хирургического лечения пациентов с углом искривления более $60^{\circ}$, крупными оссифицированными бляшками, длиной полового члена менее 13 см, наличием сложных форм деформации - являются «удлиняющие» операции. Суть данного вида оперативных пособий заключается в рассечении фиброзного участка белочной оболочки на её короткой стороне (вогнутая часть полового члена) и удлинении белочной оболочки за счёт «вставки» трансплантата из природного или синтетического материала. Ранее довольно активно проводилось иссечение бляшки, однако в последние годы подавляющее большинство хирургов от него отказались в силу высокого риска развития венозной утечки, а также - неприемлемо высокого уровня послеоперационной эректильной дисфункции $[11,26]$.

Частичное иссечение бляшки гораздо предпочтительнее полного, потому что последнее может привести к необратимой ЭД за счет нарушения веноокклюзионного механизма полового члена. Более того, послабляющее иссечение кальцинированной бляшки может быть эффективно использовано для изменения формы пениса и возможности применения трансплантата меньших размеров. Недостатком полного иссечения бляшки является тот факт, что не все пациенты имеют ощутимые бляшки на момент операции. Группы методик хирургического лечения БП с использованием различных биологических или синтетических трансплантатов показывают различные результаты, которые могут быть связаны с объемом удаленной пораженной белочной оболочки, но не материалом графта. Появившиеся литературные сообщения об увеличении темпов развития ЭД, после полного иссечения бляшки, привели к популяриза- ции только лишь рассечения бляшки и замены дефекта трансплантатом [11].

В 1991 году Gelbard и Hayden сообщили об использовании искусственной эрекции для измерения несоответствия между выпуклой и вогнутой сторонами полового члена. Авторы использовали несколько поперечных надрезов белочной оболочки, чтобы выпрямить половой член. За счет моделирования общей длины зияющих разрезов во время продольной тяги проводилась трансплантация свободного графта височной фасции, тем самым достигалась коррекция несоответствия размеров белочной оболочки [27]. Это был первый доклад, подразумевающий соответствие размера трансплантата для коррекции пенильной девиации, а также применение инцизии бляшки и графтинга. В 1995 году Gelbard сообщил об использовании двойного Y разреза, чтобы избежать уменьшения диаметра, вызванного линейными разрезами [28]. В 2002 году Egydio применил один расслабляющий разрез в точке максимальной кривизны полового члена с радиарным направлением углов до $120^{\circ}$ по типу двойного Ү-разреза. Вновь созданный дефект белочной оболочки был замещен трансплантатом, что позволило корригировать длину и сужение ствола полового члена. Автор достиг 88\% частоты коррекции полового члена у 33 больных БП, со средним увеличением длины полового члена на 2,21 см [29].

Ключевым аспектом данной группы методов хирургического лечения болезни Пейрони является выбор оптимального трансплантата. Идеальный материал для него должен быть недорогим, легкодоступным, пригодным для длительного использования, не вызывающим воспалительных и аллергических реакций, безопасным для эректильной функции и не ведущим к укорачиванию полового члена [10].

Трансплантат или графт - это материал, который используется при хирургическом лечении БП для замещения дефекта белочной оболочки при её иссечении или рассечении. «Графт»термин, подразумевающий приживление, за счет формирования новой кровеносной сети, полностью вырезанной, перемещенной, пересаженной ткани на подготовленное ложе. Процесс приживления продолжается около 96 часов и состоит из двух фаз. Начальная фаза - имбибиция, длящаяся около 48 часов, в течение которой графт (трансплантат) выживает за счет «питания» из подлежащего ложа, при этом температура графта меньше температуры организма. Вторая фаза - прививание, для которой также необходимо около 48 часов. В данный промежуток времени происходит истинное восстановление микроциркуляции графта, за счет чего тем- 
пература графта повышается до температуры тела. Процесс приживления связан, как с природой ткани графта, так и с состоянием ложа, и обусловлен васкуляризацией последнего [30].

К сожалению, «идеальный графт» все еще не определен. Это связано с отсутствием достоверных данных о правильно спланированных исследованиях по сравнению различий графтов для хирургического лечения БП. На сегодняшний день имеются публикации о создании аутологичного тканево-инженерного эндотелизированного графта, полученного путем биопсии кожи [31]. В модели на животных этот графт показал прочную силу натяжения, что адекватно сохраняет веноокклюзивный механизм. Также предпринимаются попытки создания артифициальной белочной оболочки [32].

В настоящее время в качестве трансплантатов используются следующие материалы: 1) аутотрансплантаты (дерма, венозная стенка, белочная оболочка, влагалищная оболочка яичка, слизистая оболочка щеки, фасции (широкая, височная); 2) аллотрансплантаты (трупный перикард, трупная широкая фасция, трупная твердая мозговая оболочка); 3) ксенотрансплантаты (подслизистая основа тонкой кишки свиньи, бычий перикард, дерма свиньи); 4) синтетические трансплантаты $\left(\right.$ Dacron $^{\circledR}$, Teflon ${ }^{\circledR}$, Gore-Tex ${ }^{\circledR}$ ) [11, 26, 33, 34].

Аутологичный материал подразумевает забор собственной ткани у самого реципиента. В качестве трансплантатов используют жир, кожу, влагалищную оболочку яичка, вену, слизистую щеки, широкую фасцию бедра, височную фасцию, крайнюю плоть. Аутологичные материалы имеют ряд преимуществ: вызывают меньше воспалительной реакции, имеют низкий потенциал для развития раневой инфекции по сравнению с синтетическими неаутологичными трансплантатами.

Синтетические трансплантаты в последнее время не рекомендуются, в связи с повышенным риском инфекции, аллергических реакций, воспалительных и фибротических изменений, что увеличивает частоту контрактур [3537]. Работы, которые демонстрируют удовлетворительные результаты корпопластики с применением синтетических материалов, базируются на крайне ограниченном объеме выборок (зачастую это, фактически, описания отдельных клинических случаев) [6]. Неаутологичные трансплантаты разделены на две группы: алло- и ксенотрансплантаты. В настоящее время, самые популярные ксенографты - это бычий перикард и подслизистая основа тонкой кишки свиньи. Эти два трансплантата имеют преимущество в снижении частоты осложнений, связанных с за- бором аутологичных трансплантатов, и уменьшении гипотетического риска развития инфекционных процессов, обусловленных аллотрансплантатами. Однако, свиные трансплантаты (SIS) и перикард (человеческий и бычий) не могут иметь применение у некоторых групп пациентов по религиозным или иным причинам.

Для исследования сравнивали результаты инцизии с графтингом, с использованием кожи, бычьего перикарда и подслизистой кишечника свиньи (SIS) [22, 38, 39]. Kovac J.R. и соавт. сообщили о наблюдении в течении 22 месяцев за комбинированной группой из 36 пациентов. Выпрямление полового члена в группе трупного перикарда достигнуто в 100\% случаев, в группе подслизистой кишечника свиньи (SIS) - в 77\% и с использованием дермального графта - в 60\%, с сохранением ригидности во время эрекции - в 39\%, 77\% и 60\%, соответственно [38]. Carson C.C. и соавт. сообщили об аналогичной когорте из 86 пациентов, с медианой наблюдения более 5 лет. Рецидив кривизны был выявлен у 50\% в группе дермального графта, у 24\% группы SIS и у 13\% с использованием трупного перикарда. Кроме того, во всех группах было отмечено укорочение полового члена (17-29\%) и снижение эректильной функции (средний балл МИЭФ снизился на 3-8 пунктов) [39].

Исследования последних лет, относительно результатов хирургического лечения при проведении удлиняющих операций, сосредоточены вокруг использования аутологичных и неаутологичных материалов, а именно, ксенографтов. Staerman F. и соавт. доложили об их опыте применения подслизистой тонкой кишки свиньи (SIS) у 28 пациентов. Несмотря на ранние, вполне удовлетворительные результаты, у 33\% больных развился рецидив искривления. Авторы связали такой исход с реактивными свойствами самого графта SIS, что побудило их сделать заключение - пациенты должны быть предупреждены о риске рецидива искривления [40].

Simonato A . и соавт. описали результаты использования пенильного кожного лоскута с периодом наблюдения 95 месяцев, в которых достигли удовлетворенности пациентов в $41 \%$ и развившейся послеоперационной ЭД в 32\% случаев. Следует отметить, что у $41 \%$ пациентов, из так называемой неудовлетворенной группы, в среднем через 8 месяцев имелась кистообразная трансформация в зоне хирургического вмешательства. Этим пациентам было рекомендовано оперативное вмешательство по удалению этих кист. В соответствии с полученными результатами исследования, авторы не рекомендуют использование кожного лоскута для замещения дефектов белочной оболочки [41]. 
B другом исследовании Da Ros C.T. и соавт. описали отдаленные результаты использования аутологичной ткани - белочной оболочки ножек полового члена. Авторы сами отметили неблагоприятные результаты - развитие послеоперационной ЭД и такие факторы риска, как травматическая мобилизация сосудисто-нервного пучка, нарушение гемодинамики полового члена, за счет уменьшения диаметра его ножек [21].

Рецидив искривления после успешно выполненного оперативного вмешательства может развиться в 10-33\% случаев, особенно, когда встречаются новые случаи БП $[36,40]$. Общий уровень неудовлетворительных функциональных результатов и определенная тенденция их нарастания в долгосрочной перспективе обусловливают необходимость разработки комплекса тщательного послеоперационного восстановительного лечения, а также длительного амбулаторного сопровождения данной группы пациентов. Более того, утверждается, что риск развития ЭД в постоперационном периоде зачастую напрямую связан с наличием этого расстройства до операции [42]. Таким образом, негативные моменты для группы удлиняющих методов связаны с высокими рисками развития эректильной дисфункции (по некоторым данным, это осложнение развивается у $25 \%$ пациентов) и повторного оперативного вмешательства на протяжении последующих 10 лет (такой риск составляет около 17\%) [35]. Отметим, что один из явных путей снижения риска повторной операции - это использование биологически более приемлемых аутотрансплантатов.

Данная группа методов несколько «проигрывает» пликациям по длительности и сложности хирургических вмешательств, но удлиняющие методики являются предпочтительным способом хирургического лечения БП. Один из наиболее весомых факторов, подтверждающих наше суждение - это отсутствие укорочения полового члена, как результата хирургического вмешательства [26, 42].

Подводя итоги вышеизложенного, можно констатировать, что каждая группа методов хирургического лечения болезни Пейрони имеет свои преимущества и недостатки. Немаловажное значение имеет и опыт оперирующего хирурга, характер деформации и исходное состояние пациента.

\section{З АК ЛЮЧ Е ИЕ}

Хирургическое лечение остается методом выбора лечения БП. На выбор метода оперативного лечения влияет угол искривления, длина полового члена, эректильный статус пациента. Учитывая развитие укорочения полового члена после пликационных методик, методом выбора являются удлиняющие операции. Из вышеизложенного литературного обзора, следует, что поиск наиболее благоприятного трансплантата для замещения белочной оболочки является весьма актуальной и малоизученной проблемой. С другой стороны, малоизученными являются отдаленные результаты использования самих графтов, которые имеют риски формирования контрактуры и связанных с этим рецидивов искривления и укорочения полового члена, развития эректильной дисфункции и в последующем - психологических проблем.

\section{Ю.Ю. Малинин, С.Г. Ермилов, Г.И. Ермилов}

ГОО ВПО «Донецкий национальный медицинский университет имени М. Горького», Донецк

\section{ХИРУРГИЧЕСКИЕ МЕТОДЫ ЛЕЧЕНИЯ БОЛЕЗНИ ПЕЙРОНИ}

Хирургическое лечение болезни Пейрони на сегодняшний день остается наиболее надежным и быстрым способом устранения девиации полового члена. Несмотря на значительные успехи реконструктивной хирургии полового члена при болезни Пейрони, анализ отечественных и зарубежных источников литературы свидетельствует о неудовлетворительных результатах хирургического лечения, поскольку осложнения и рецидивы болезни, как и раньше, остаются на достаточно высоком уровне - от $10 \%$ до $70 \%$ наблюдений. До настоящего времени не существует «золотого стандарта» в хирургическом подходе. Однако главной целью хирургического лечения является выпрямление полового члена и восстановление проникающей способности для адекватного проведения полового акта. Решающими факторами при выборе того или иного метода хирургической коррекции являются угол искривления, тип деформации, длина пениса, состояние эректильной функции, а также ожидания пациента и полового партнера. Устранить искривление полового члена без его укорочения позволяют пластические операции: заместительная корпоропластика или графтинг, а дефект белочной оболочки после иссечения бляшки закрывают трансплантатом. Ключевым аспектом данной группы методов хирургического лечения болезни Пейрони является выбор оптимального трансплантата. Идеальный материал для него должен быть недорогим, легкодоступным, пригодным для длительного использования, не вызывающим воспалительных и аллергических реак- 
ций, безопасным для эректильной функции и не ведущим к укорачиванию полового члена. K сожалению, «идеальный трансплантат» для заместительной корпоропластики все еще не определен.
Ключевые слова: болезнь Пейрони, пликация, корпоропластика, трансплантат.

\section{Yu.Yu. Malinin, S.G. Ermilov, G.I. Ermilov}

\section{SEI HPE «M. Gorky Donetsk National Medical University», Donetsk}

\section{SURGICAL TREATMENT OF PEYRONIE'S DISEASE}

Surgical treatment of Peyronie's disease remains today the most reliable and fastest way to eliminate penile deviation. Despite the significant success of reconstructive surgery of the penis in Peyronie's disease, the analysis of native and foreign literature sources indicates unsatisfactory results of surgical treatment, since complications and relapses of the disease, as before, remain at a fairly high level - from $10 \%$ to $70 \%$ of observations. Until now, there is no «gold standard» in the surgical approach. However, the main goal of surgical treatment is to straighten the penis and restore penetrating ability for adequate sexual intercourse. The decisive factors when choosing one or another method of surgical correction are the angle of curvature, the type of deformity, the length of the penis, the state of erectile function, as well as the expectations of the patient and sexual partner. Plastic surgery allows to eliminate the curvature of the penis without shortening it: corporoplasty replacement or grafting, and the defect in the tunica albuginea after excision of the plaque is closed with a graft. The key aspect of this group of surgical methods for the treatment of Peyronie's disease is the selection of the optimal graft. The ideal material for it should be inexpensive, easily accessible, suitable for long-term use, does not cause inflammatory and allergic reactions, safe for erectile function and does not lead to shortening of the penis. Unfortunately, the "perfect transplant" for corporoplasty replacement surgery has not yet been determined.

Key words: Peyronie's disease, plication, corporoplasty, transplant.

\section{ЛИТЕРАТУРА}

1. Малей М. Франсуа Пейрони - лейб-медик короля, заложивший фундамент будущего урологии. Медицинские аспекты здоровья мужчин. 2014; 4 (15): 61-63.

2. Sommer F. Epidemiology of Peyronie's disease. Int. J. Impotence res. 2002; 14: 379-383.

3. Mulhall J.P., Schiff J., Guhring P. An analysis of the natural history of Peyronie's disease. J. Urol. 2006; 75 (6): 2115-8.

4. Гамидов С.И., Попков В.М., Шытылко Т.В., Овчинников Р.И., Королев А.Ю. и соавт. Отдаленные результаты корпоропластики при болезни Пейрони. Андрология и генитальная хирургия. 2018; 4: 39-45.

5. Москалева Ю.С., Корнеев И.А. Результаты хирургического лечения при болезни Пейрони. Урологические ведомости. 2017; 7 (1): 25-29.

6. Болезнь Пейрони. Под ред. проф. П.А. Щеплева. М.: ИД «АБВ-пресс»; 2012. 216

7. Kadioglu A., Akman T., Sanli O. et al. Surgical treatment of Peyronie's disease: a critical analysis. Eur .Urol. 2006; 50 (2): 235-48.

8. Карагужин С.К. Современный подход к лечению фибропластической индурации полового члена: автореферат дисс. док. мед. наук. Москва; 2010. 36.

9. Горпинченко И.И., Романюк М.Г. Коллост - уникальный биопластический материал. Перспективы в андрологии. Здоровье мужчины. 2009; 1 (28):83-86.

10. Кызласов П.С., Мартов А.Г., Помешкин Е.В. и соавт. Лечение болезни Пейрони. Медицина в Кузбассе. 2017; $1: 3-10$.

11. Москалева Ю.С., Остапченко А.Ю., Корнеев И.А. Болезнь Пейрони. Урологические ведомости. 2015; 4: 30-35.

12. Гамидов С.И., Овчинников Р.И., Тажетдинов О.Х., Павловичев А.А. Консервативное и хирургическое лечение болезни Пейрони. Фарматека. 2010; 18: 49-54.

13. Даренков С.П., Саркисян А.Д. Оперативное лечение болезни Пейрони с использованием аутологичных материаловенков. Кремлевская медицина. Клинический

\section{REFERENCES}

1. Malej M. Fransua Pejroni - lejb-medik korolja, zalozhivshij fundament budushhego urologii. Medicinskie aspekty zdorov'ja muzhchin. 2014; 4 (15): 61-63. (in Russian).

2. Sommer F. Epidemiology of Peyronie's disease. Int. J. Impotence res. 2002; 14: 379-383.

3. Mulhall J.P., Schiff J., Guhring P. An analysis of the natural history of Peyronie's disease. J. Urol. 2006; 75 (6): 2115-8.

4. Gamidov S.I., Popkov V.M., Shytylko T.V., Ovchinnikov R.I., Korolev A.Ju. i soavt. Otdalennye rezul'taty korporoplastiki pri bolezni Pejroni. Andrologija i genital'naja hirurgija. 2018; 4: 39-45. (in Russian).

5. Moskaleva Ju.S., Korneev I.A. Rezul'taty hirurgicheskogo lechenija pri bolezni Pejroni. Urologicheskie vedomosti. 2017; 7 (1): 25-29. (in Russian).

6. Bolezn’ Pejroni. Pod red. prof. P.A. Shhepleva. M.: ID «ABVpress»; 2012. 216. (in Russian).

7. Kadioglu A., Akman T., Sanli O. et al. Surgical treatment of Peyronie's disease: a critical analysis. Eur .Urol. 2006; 50 (2): 235-48.

8. Karaguzhin S.K. Sovremennyj podhod k lecheniju fibroplasticheskoj induracii polovogo chlena: avtoreferat diss. dok. med. nauk. Moskva; 2010. 36. (in Russian).

9. Gorpinchenko I.I., Romanjuk M.G. Kollost - unikal'nyj bioplasticheskij material. Perspektivy v andrologii. Zdorov'e muzhchiny. 2009; 1 (28):83-86. (in Russian).

10. Kyzlasov P.S., Martov A.G., Pomeshkin E.V. i soavt. Lechenie bolezni Pejroni. Medicina v Kuzbasse. 2017; 1: 3-10. (in Russian).

11. Moskaleva Ju.S., Ostapchenko A.Ju., Korneev I.A. Bolezn' Pejroni. Urologicheskie vedomosti. 2015; 4: 30-35. (in Russian).

12. Gamidov S.I., Ovchinnikov R.I., Tazhetdinov O.H., Pavlovichev A.A. Konservativnoe i hirurgicheskoe lechenie bolezni Pejroni. Farmateka. 2010; 18: 49-54. (in Russian).

13. Darenkov S.P., Sarkisjan A.D. Operativnoe lechenie bolezni Pejroni s ispol'zovaniem autologichnyh materialovenkov. Kremlevskaja medicina. Klinicheskij vestnik. 2017; 2: 
вестник. 2017; 2: 65-70.

14. Малышев В.А., Кунильский С.Е., Малышев Е.В. Опыт хирургического лечения эректильной деформации полового члена. Урологические ведомости. 2015; 1: 45.

15. Chen R., McCraw C., Lewis R. Plication procedures - excisional and incisional corporoplasty and imbrication for Peyronie's disease. Transl. Androl. Urol. 2016; 5 (3): 31833.

16. Pryor J., Akkus E., Alter G. et al. Peyronie’s disease. J. Sex. Med. 2004; l (1): 110-5.

17. Polkey H.J. Induratio penis plastic. Urol. Cut. Rev. 1928; 32: 287-308

18. Nesbit R.M. Congenital curvature of the phallus: report of three cases with description of corrective operation. J. Urol. 1965; 93: 230-232.

19. Pryor J.P., Fitzpatrick J .M. A new approach to the correction of the penile deformity in Peyronie's disease. J. Urol. 1979; 122 (5): 622-623.

20. Martenstein C., Peruth J., Hamza A. The role of Nesbit's procedure in surgical reconstruction of penile deviation. GMS Interdiscip. Plast. Reconstr. Surg. DGPW. 2012; 1: $1-6$.

21. Da Ros C.T., Graziottin T. M., Ribeiro E. et al. Longterm follow-up of penile curvature correction utilizing autologous albugineal crural graft. Int. Braz. J. Urol. 2012; 38 (2): 242-247.

22. Mandava S.H., Trost L.W., Hellstrom W.J. Critical analysis of the surgical outcomes for the treatment of Peyronie's disease. Arab. J. Urol. 2013; 11 (3): 284-293.

23. Sassine A.M., Wespes E., Schulman C.C. Modified corporoplasty for penile curvature: 10 years' experience. Urology.1994; 44 (3): 321-419.

24. Adibi M., Hudak S.J., Morey A.F. Penile plication wi thout degloving enables effective correction of complex Peyronie's deformities. Urology. .2012; 79 (4): 831-835.

25. Parker J., Martinez D., Hakky T. et al. Minimally invasive modification of the dot plication technique utilizing absorbable sutures. J. Sex. Med. 2012; 9 (4):183-298.

26. Segal R.L., Burnett A.L. Surgical management for Peyronie's disease. World J. Mens. Health. 2013; 31: 1-11.

27. Gelbard M.K., Hayden B. Expanding contractures of the tunica albuginea dueto Peyronie's disease with temporalis fascia free grafts. J. Urol. 1991; 145 (4): 772-776.

28. Gelbard M. K. Relaxing incisions in the correction of penile deformity due to Peyronie's disease. J. Urol. 1995; 154 (4): 1457-1460.

29. Egydio P.H., Lucon A. M., Arap S. Treatment of Peyronie's disease by incomplete circumferential incision of the tunica albuginea and plaque with bovine pericardium graft. Urology. 2002; 59 (4): 570-574.

30. Kakonashivili A.P., Shioshvili T.J. Substitution of tunica albuginea penis by different autotransplant: an experimental study. Georgian Med. News. 2003; 10: 38-42.

31. Imbeault A., Bernard G., Ouellet G. et al. Surgical option for the correction of Peyronie's disease: a nautologous tissue-engineered endothelialized graft. J. Sex. Med. 2011; 8 (11): 3227-3235.

32. Ferretti L., Giuliani M., Bessède T., et al. Tissue engineering f or penile surgery: comparative study of noncellular and cell-seeded synthetic grafts f or tunica albuginea replacement. J. Sex. Med. 2012; 9 (2): 625- 631.

33. Garaffa G., Trost L.W., Serefoglu E. C. et al. Understanding the course of Peyronie's. Int. J. Clin. Pract. 2013; 67 (8): $781-788$.

34. Zaid U.B., Alwaal A., Zhang X. et al. Surgical Management of Peyronie's Disease. Curr. Urol. Rep. 2014; 15 (10): 446.

35. Kadioglu A., Akman T., Sanli O. et al. Surgical treatment of Peyronie's disease: a critical analysis. Eur .Urol. 2006; 50 (2): 235-248.

36. Levine L.A., Burnett A. L. Standard Operating Procedures for Peyronie's Disease. J. Sex. Med. 2013; 10 (1): 230-244.

37. Martinez D., Ercole C.E., Hakky T.S. et al. Peyronie's Disease: still a surgical disease. Advances in Urology. 2012; 20 (1): 5 .

38. Kovac J. R. Brock G.B. Surgical outcomes and patient satisfaction after dermal, pericardial, and small intestinal
65-70. (in Russian).

14. Malyshev V.A., Kunil'skij S.E., Malyshev E.V. Opyt hirurgicheskogo lechenija jerektil'noj deformacii polovogo chlena. Urologicheskie vedomosti. 2015; 1: 45. (in Russian).

15. Chen R., McCraw C., Lewis R. Plication procedures - excisional and incisional corporoplasty and imbrication for Peyronie's disease. Transl. Androl. Urol. 2016; 5 (3): 31833.

16. Pryor J., Akkus E., Alter G. et al. Peyronie's disease. J. Sex. Med. 2004; l (1): 110-5.

17. Polkey H.J. Induratio penis plastic. Urol. Cut. Rev. 1928; 32: 287-308.

18. Nesbit R.M. Congenital curvature of the phallus: report of three cases with description of corrective operation. J. Urol. 1965; 93: 230-232.

19. Pryor J.P., Fitzpatrick J .M. A new approach to the correction of the penile deformity in Peyronie's disease. J. Urol. $1979 ; 122$ (5): 622-623.

20. Martenstein C., Peruth J., Hamza A. The role of Nesbit's procedure in surgical reconstruction of penile deviation. GMS Interdiscip. Plast. Reconstr. Surg. DGPW. 2012; 1: 1-6.

21. Da Ros C.T., Graziottin T. M., Ribeiro E. et al. Longterm follow-up of penile curvature correction utilizing autologous albugineal crural graft. Int. Braz. J. Urol. 2012; 38 (2): 242-247.

22. Mandava S.H., Trost L.W., Hellstrom W.J. Critical analysis of the surgical outcomes for the treatment of Peyronie's disease. Arab. J. Urol. 2013; 11 (3): 284-293.

23. Sassine A.M., Wespes E., Schulman C.C. Modified corporoplasty for penile curvature: 10 years' experience. Urology.1994; 44 (3): 321-419.

24. Adibi M., Hudak S.J., Morey A.F. Penile plication wi thout degloving enables effective correction of complex Peyronie's deformities. Urology. .2012; 79 (4): 831-835.

25. Parker J., Martinez D., Hakky T. et al. Minimally invasive modification of the dot plication technique utilizing absorbable sutures. J. Sex. Med. 2012; 9 (4):183-298.

26. Segal R.L., Burnett A.L. Surgical management for Peyronie's disease. World J. Mens. Health. 2013; 31: 1-11.

27. Gelbard M.K., Hayden B. Expanding contractures of the tunica albuginea dueto Peyronie's disease with temporalis fascia free grafts. J. Urol. 1991; 145 (4): 772-776.

28. Gelbard M. K. Relaxing incisions in the correction of penile deformity due to Peyronie's disease. J. Urol. 1995; 154 (4): 1457-1460.

29. Egydio P.H., Lucon A. M., Arap S. Treatment of Peyronie's disease by incomplete circumferential incision of the tunica albuginea and plaque with bovine pericardium graft. Urology. 2002; 59 (4): 570-574.

30. Kakonashivili A.P., Shioshvili T.J. Substitution of tunica albuginea penis by different autotransplant: an experimental study. Georgian Med. News. 2003; 10: 38-42. (in Russian).

31. Imbeault A., Bernard G., Ouellet G. et al. Surgical option for the correction of Peyronie's disease: a nautologous tissue-engineered endothelialized graft. J. Sex. Med. 2011; 8 (11): 3227-3235.

32. Ferretti L., Giuliani M., Bessède T., et al. Tissue engineering f or penile surgery: comparative study of noncellular and cell-seeded synthetic grafts $f$ or tunica albuginea replacement. I. Sex. Med. 2012; 9 (2): 625-631.

33. Garaffa G., Trost L.W., Serefoglu E. C. et al. Understanding the course of Peyronie's. Int. J. Clin. Pract. 2013; 67 (8): 781- 788.

34. Zaid U.B., Alwaal A., Zhang X. et al. Surgical Management of Peyronie's Disease. Curr. Urol. Rep. 2014; 15 (10): 446.

35. Kadioglu A., Akman T., Sanli O. et al. Surgical treatment of Peyronie's disease: a critical analysis. Eur .Urol. 2006; 50 (2): 235-248.

36. Levine L.A., Burnett A. L. Standard Operating Procedures for Peyronie's Disease. J. Sex. Med. 2013; 10 (1): 230-244.

37. Martinez D., Ercole C.E., Hakky T.S. et al. Peyronie's Disease: still a surgical disease. Advances in Urology. 2012; $20(1): 5$. 
submucosal grafting for Peyronie's disease. J. Sex. Med. 2007; 4 (5): 1500-1508.

39. Carson, C.C., Levine L.A. Outcomes of surgical treatment of Peyronie's disease. BJU Int. 2014; 113 (5): 704-713.

40. Staerman F., Pierrevelcin J., Ripert T. et al. Medium-term follow-up of plaque incision and porcine small intestinal submucosal grafting for Peyronie's disease. Int. J. Impot. Res. 2010; 22 (6): 343-348.

41. Simonato A ., Gregori A., Varca V. et al. Penile dermal f lap in patients with Peyronie's disease: long- term results. J. Urol. 2010; 183 (3): 1065-1068.

42. Levine L. A., Goldman K. E., Greenfield J. M. Experience with intraplaque injection of verapamil for Peyronie's disease. J. Urol. 2002; 168: 621-625.
38. Kovac J. R. Brock G.B. Surgical outcomes and patient satisfaction after dermal, pericardial, and small intestinal submucosal grafting for Peyronie's disease. J. Sex. Med. 2007; 4 (5): 1500-1508.

39. Carson, C.C., Levine L.A. Outcomes of surgical treatment of Peyronie’s disease. BJU Int. 2014; 113 (5): 704-713.

40. Staerman F., Pierrevelcin J., Ripert T. et al. Medium-term follow-up of plaque incision and porcine small intestinal submucosal grafting for Peyronie's disease. Int. J. Impot. Res. 2010; 22 (6): 343-348.

41. Simonato A., Gregori A., Varca V. et al. Penile dermal f lap in patients with Peyronie's disease: long- term results. J. Urol. 2010; 183 (3): 1065-1068.

42. Levine L. A., Goldman K. E., Greenfield J. M. Experience with intraplaque injection of verapamil for Peyronie's disease. J. Urol. 2002; 168: 621-625. 